\title{
Three-dimensional echocardiography-guided atrial septectomy: An experimental study
}

\author{
Yoshihiro Suematsu, MD ${ }^{\mathrm{a}, \mathrm{c}}$ \\ Gerald R. Marx, MD ${ }^{\mathrm{a}}$ \\ John K. Triedman, $M D^{a}$ \\ Tomislav Mihaljevic, $\mathrm{MD}^{\mathrm{b}}$ \\ Bassem N. Mora, MD \\ Shinichi Takamoto, MD ${ }^{\mathrm{C}}$ \\ Pedro J. del Nidoa
}

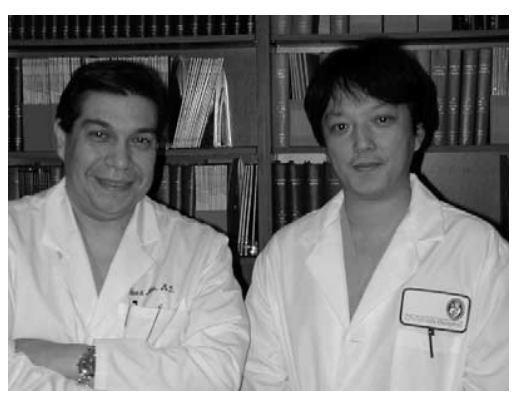

Drs del Nido and Suematsu
From the Departments of Cardiac Surgery and Cardiology, Children's Hospital-Boston, ${ }^{\mathrm{a}}$ and the Division of Cardiac Surgery, Brigham and Women's Hospital, Harvard Medical School, ${ }^{\mathrm{b}}$ Boston, Mass, and the Division of Cardiothoracic Surgery, University of Tokyo, Tokyo, Japan. ${ }^{\mathrm{c}}$

Supported in part by National Institutes of Health grants HL71128 and HL 73647 (P.J.D.) and by the Japan Society for the Promotion of Science (Y.S.).

Received for publication Jan 23, 2004; revisions requested March 8, 2004; accepted for publication March 11, 2004

Address for reprints: Pedro J. del Nido, MD, Department of Cardiac Surgery, Children's Hospital-Boston, 300 Longwood Ave, Boston, MA 02115 (E-mail: pedro. delnido@tch.harvard.edu).

J Thorac Cardiovasc Surg 2004;128:53-9

$0022-5223 / \$ 30.00$

Copyright $\odot 2004$ by The American Association for Thoracic Surgery

doi:10.1016/j.jtcvs.2004.03.008
Objectives: The surgical feasibility of beating-heart atrial septectomy under the guidance of two different types of real-time 3-dimensional echocardiography systems was examined.

Methods: A modified real-time 3-dimensional echocardiography system with a $\times 4$ matrix transducer (Sonos 7500; Philips Medical Systems, Andover, Mass) or a mechanical 1-dimensional array transducer (SSD-5500; Aloka Co, Ltd, Tokyo, Japan) was used. Small porcine atrial septal defects $(n=8)$ were enlarged with a Kerrison bone punch in the tank model. In the animal studies, small atrial septal defects $(n=8)$ were enlarged with the same device through a transatrial port. In both experiments, the area of the atrial septal defect measured by real-time 3-dimensional echocardiography was compared with the area measured directly.

Results: Real-time 3-dimensional echocardiography provided satisfactory images and sufficient anatomic detail for the atrial septectomy in both experimental settings. All the atrial septal defects were successfully enlarged; an increase of as much as $293 \%$ of the preoperative atrial septal defect area was achieved in the tank experiment, and an increase of as great as $449 \%$ of the preoperative area was achieved in the animal experiment. The size of the atrial shunt was increased significantly after the atrial septectomy relative to that before the surgery $(P<.0001)$. The percentage enlargement of the atrial septal defect measured by real-time 3-dimensional echocardiography was strongly correlated with that measured directly (both $r^{2}=0.997$, $P<.0001)$ Bland-Altman analysis showed close agreement between the results obtained by the two measurement methods in both models.

Conclusions: Real-time 3-dimensional echocardiography provides satisfactory images and sufficient anatomic detail for atrial septectomy. This experiment demonstrates the surgical feasibility of a beating-heart intracardiac procedure such as atrial septectomy under real-time 3-dimensional echocardiographic guidance.

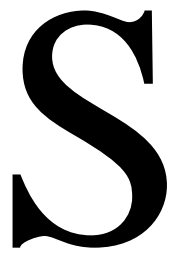

urgical septectomy is essential for the palliation of some types of complex congenital cardiac anomalies, such as transposition of the great arteries and left-sided atrioventricular valve stenosis or atresia ${ }^{1}$. In such cases balloon atrial septostomy has been conventionally attempted since the first introduction of this method by Rashkind and Miller in $1966 .{ }^{2}$ However, balloon atrial septostomy is not always effective or adequate, especially in older infants with a thickened atrial septum. ${ }^{3}$ The Blalock-Hanlon procedure has been conducted to create an atrial septal defect (ASD), but performance of this procedure requires expert technical skill and carries the risks of significant blood loss and air embolization. ${ }^{4}$ Surgical atrial septectomy 


\section{D Matrix Array Mechanical 1D Array}

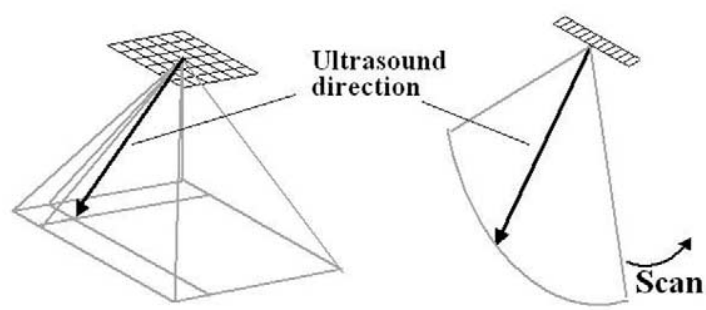

Figure 1. Schematic diagram illustrating principles of two different RT3DE systems. Left, Full volume-rendering system with 2-dimensional (2D) matrix array transducer. Right, High-speed volume-rendering system with mechanical 1-dimensional (1D) microconvex array transducer.

with cardiopulmonary bypass is the most effective procedure for establishing controlled interatrial communication; however, cardiopulmonary bypass itself is a major intervention, especially in critically ill patients.

Real-time 3-dimensional echocardiography (RT3DE) has recently been developed and provides clinicians and surgeons with a new tool for immediate visualizing the heart noninvasively. ${ }^{5}$ This new method has great potential for expanded application, not only in the area of medical diagnosis but also for image-guided surgical interventions. In this study we examined the surgical feasibility of beatingheart atrial septectomy under the guidance of two different RT3DE systems, one used in a tank experiment and the other in an animal experiment.

\section{Materials and Methods}

\section{Echocardiographic Equipment}

In the tank study, RT3DE was performed with the $\times 4$ matrix transducer on a Sonos 7500 system (Philips Medical Systems, Andover, Mass). The transducer operates over a broadband range of 2 to $4 \mathrm{MHz}$ and scans a 3-dimensional volume by electronically steering the acoustic beam with a matrix of approximately 3000 transducer elements and associated electronics that allows scanning of a $64^{\circ} \times 64^{\circ}$ pyramidal volume in real time as fast as 28 frames/s (Figure 1, left). This system volume renders data at any desired viewing orientation, and the orientation of the target object on the screen can be controlled with a roller ball.

In the animal studies, the Sonos 7500 system was not found to be suitable for epicardial use because of the large probe size and low frequency range of the transducer. RT3DE was therefore performed with a high-speed 3-dimensional rendering unit installed in the ultrasonographic equipment SSD-5500 (Aloka Co, Ltd, Tokyo, Japan) and a special transducer for acquiring 3-dimensional echocardiographic data. The 3-dimensional transducer consists of a microconvex array transducer with a 6-MHz center frequency and a motor that mechanically moves the array transducer to transmit ultrasonic waves and receive echocardiographic data from 3-dimensional space. An ultrasound beam transmitted

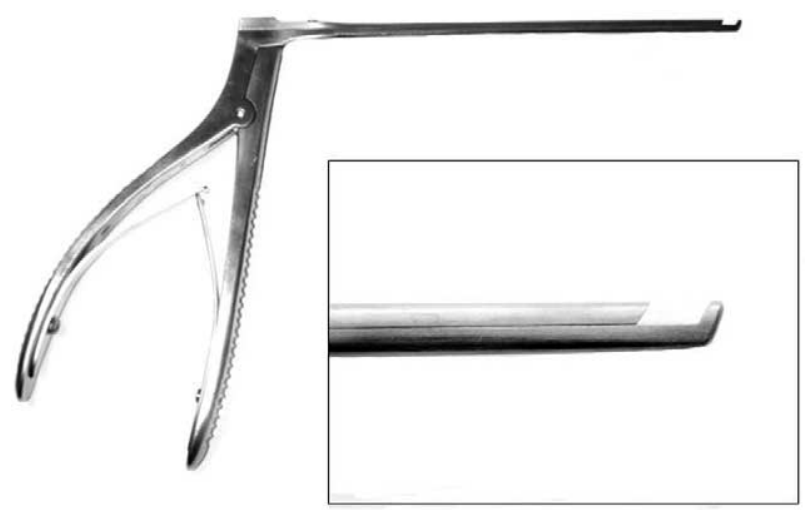

Figure 2. Kerrison bone punch.

from the transducer is scanned both electrically and mechanically within a $45^{\circ} \times 45^{\circ}$ area (Figure 1 , right). The frame rate of the 3-dimensional images rendered by RT3DE changes from 5 to 10 frames/s, depending on the size of the area and the image resolution desired.

\section{Tank Study}

A custom tank was prepared for evaluating the performance of the surgical procedure under simulated clinical image-guidance conditions. This tank consisted of an acrylic reservoir covered by an opaque dome through which surgical instruments could be inserted. The bottom of the tank was lined with an acoustic polymer (Shin-Etsu Polymer Co, Ltd, Tokyo, Japan) to prevent echo beam reflections from the tank. Degassed double-deionized water was used as the imaging medium inside the testing tank.

Interatrial septa from 8 slaughterhouse pig hearts were mounted in the water tank, and excision was made in the septum secundum region to create a small ASD. Then, under RT3DE guidance, the ASD was enlarged with a Kerrison bone punch (Figure 2). During the experiment, the operator was blinded to the actual target and operated only according to ultrasonographic guidance. Real-time images were displayed on a television monitor and recorded on a standard video recorder. Both macroscopic ASD images photographed with a digital camera and ASD images recorded by RT3DE were digitized onto a personal computer (Sony Corp, Tokyo, Japan), and planimetric measurements of the echocardiographic recordings were conducted with Scion Image software (Scion Corp, Gaithersburg, Md). The sizes of the ASD before and after the atrial septectomy were compared by comparison of the macroscopic images, calculation of the percentage enlargement of the ASD (postseptectomy ASD size/preseptectomy ASD size $\times$ $100 \%$ ) in the RT3DE images (\%ERT3DE), and calculation from direct measurements (\%EDM).

\section{Animal Study}

The study protocol was approved by a review committee of the University of Tokyo. All animals received humane care in compliance with the "Guidelines for the Care and Use of Laboratory Animals" (http://www.nap.edu/catalog/5140.html) and with the approval of the University of Tokyo Institutional Animal Care and Use Committee. 
A

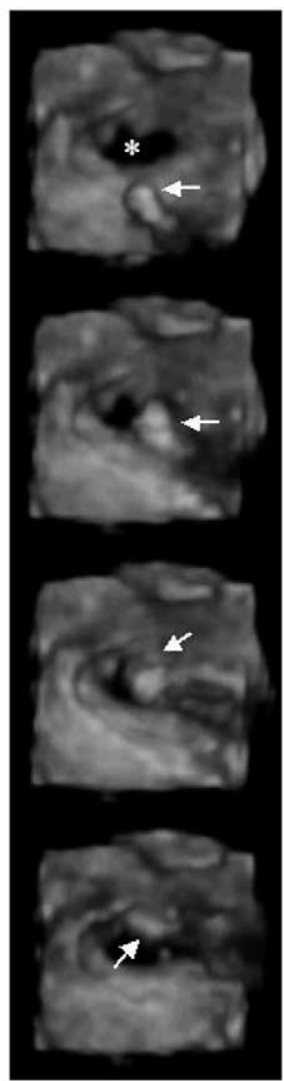

E

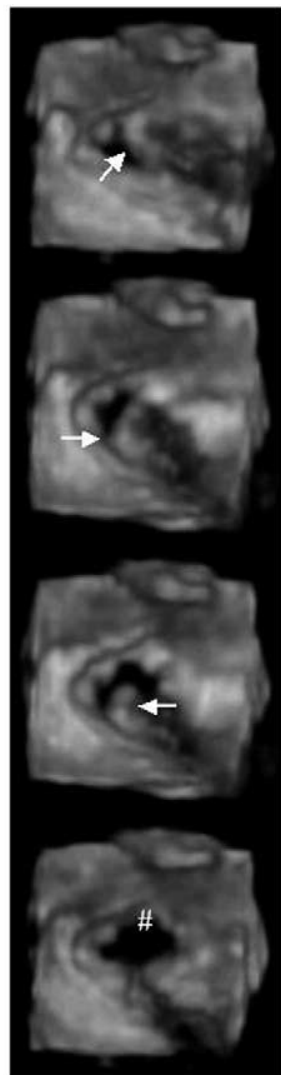

Figure 3. RT3DE images obtained in tank study showing atrial septectomy with Kerrison bone punch. Asterisk denotes ASD; white arrow indicates tip of Kerrison bone punch; crosshatch denotes area of ASD enlarged by atrial septectomy.

Eight mongrel dogs (9.8-14.5 kg) were anesthetized with nembutal $(30 \mathrm{mg} / \mathrm{kg}$ intravenously), and anesthesia was maintained with $2 \%$ isoflurane. Respiration was maintained with a volumecontrol respirator. Continuous electrocardiographic monitoring was conducted throughout the procedure. Arterial blood gas values were determined every 30 minutes, and bicarbonate was administered as needed to maintain the physiologic $\mathrm{pH}$ between 7.35 and 7.45. Aortic blood pressure was monitored with a $5 \mathrm{~F}$ micromanometer-tipped catheter (Millar Instruments, Houston, Tex) introduced through the left femoral artery.

A median sternotomy was performed, the right atrium was suspended upward with sutures, and the echo probe was applied directly to the surface of the right atrium. A purse-string suture of 4-0 polypropylene was placed around the right atrial appendage. Surgical atrial septectomy was performed through a dilating-tip trocar (Ethicon, Inc, Somerville, NJ) inserted through the pursestring suture after intravenous administration of heparin (100 $\mathrm{U} / \mathrm{kg}$ ). While the devices were inserted, blood was slowly drawn through the side hole of the trocar to prevent introduction of air into the cardiac cavity. Under RT3DE guidance, a balloon catheter was inserted, and the balloon was inflated to create a small ASD within the fossa ovalis. This ASD was then enlarged with a Kerrison bone punch; the maximum diameters of the ASD before
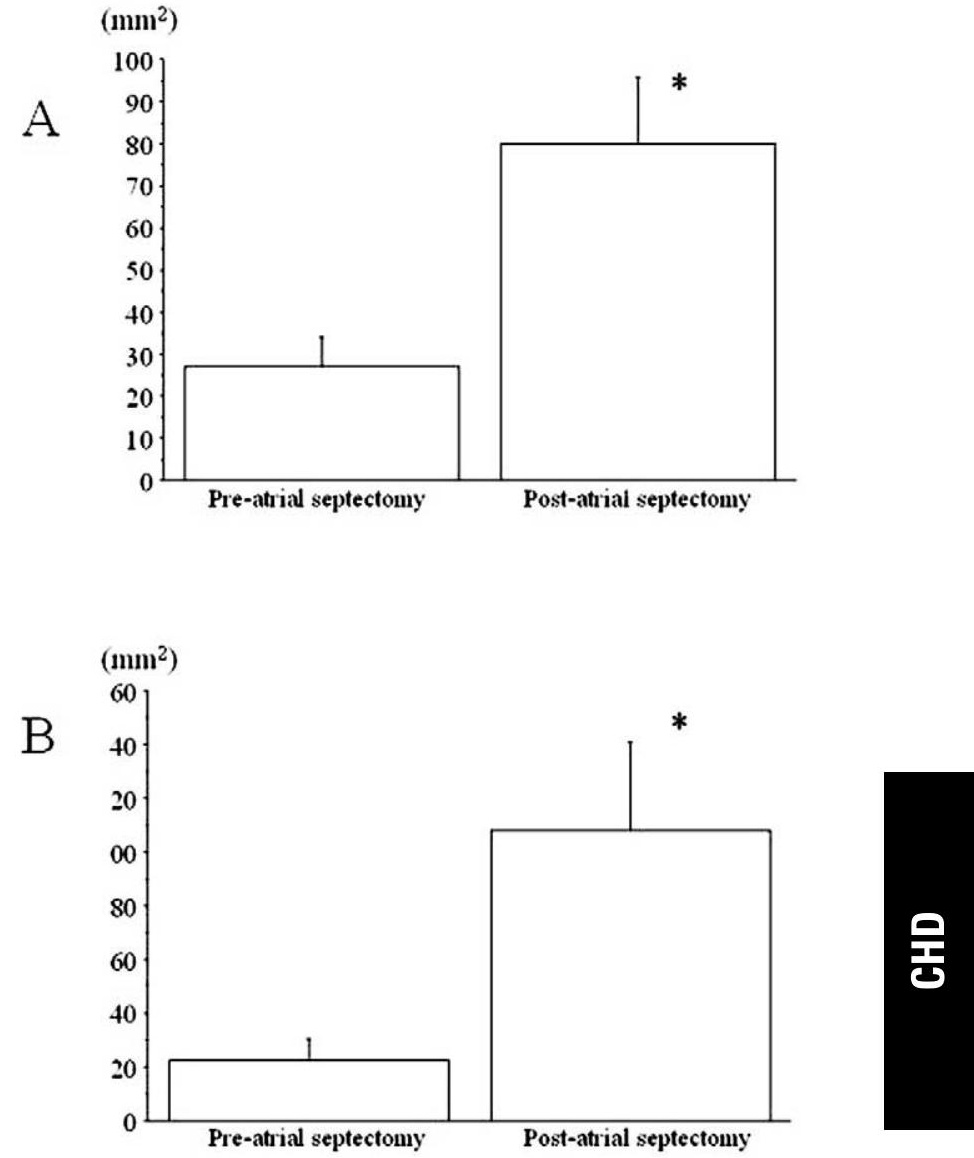

Figure 4. A, Size comparison of ASD before and after atrial septectomy in macroscopic ASD images obtained in tank study. B, Size comparison of ASD before and after atrial septectomy from ASD images obtained by RT3DE in animal study. Significant differences $(P=.05)$ versus ASD before atrial septectomy are designated by asterisks.

and after the atrial septectomy were then calculated from the real-time 3-dimensional images.

The sizes of the atrial shunt before and after the atrial septectomy were graded by 2-dimensional epicardial echocardiography according to the following scale: 1 , none, no color Doppler disturbance on the right atrial side; 2 , trivial, minimal color disturbance on the right atrial side $(<1 \mathrm{~mm}$ wide at the origin of the color Doppler jet); 3, small, 1 to $2 \mathrm{~mm}$ wide at the origin of the color Doppler jet; 4, moderate, more than $2 \mathrm{~mm}$ wide at the origin of the color Doppler jet; and 5, large, large or multiple color jets.

Macroscopic ASD images obtained with a digital camera just after the heart was excised and ASD images recorded by RT3DE before and after the atrial septectomy were digitized, and planimetric measurements of the echocardiographic recordings were conducted as described for the tank experiments. The sizes of the ASD before and after atrial septectomy were compared in the ASD images obtained by RT3DE, and the \%ERT3DE and \%EDM were then compared. 

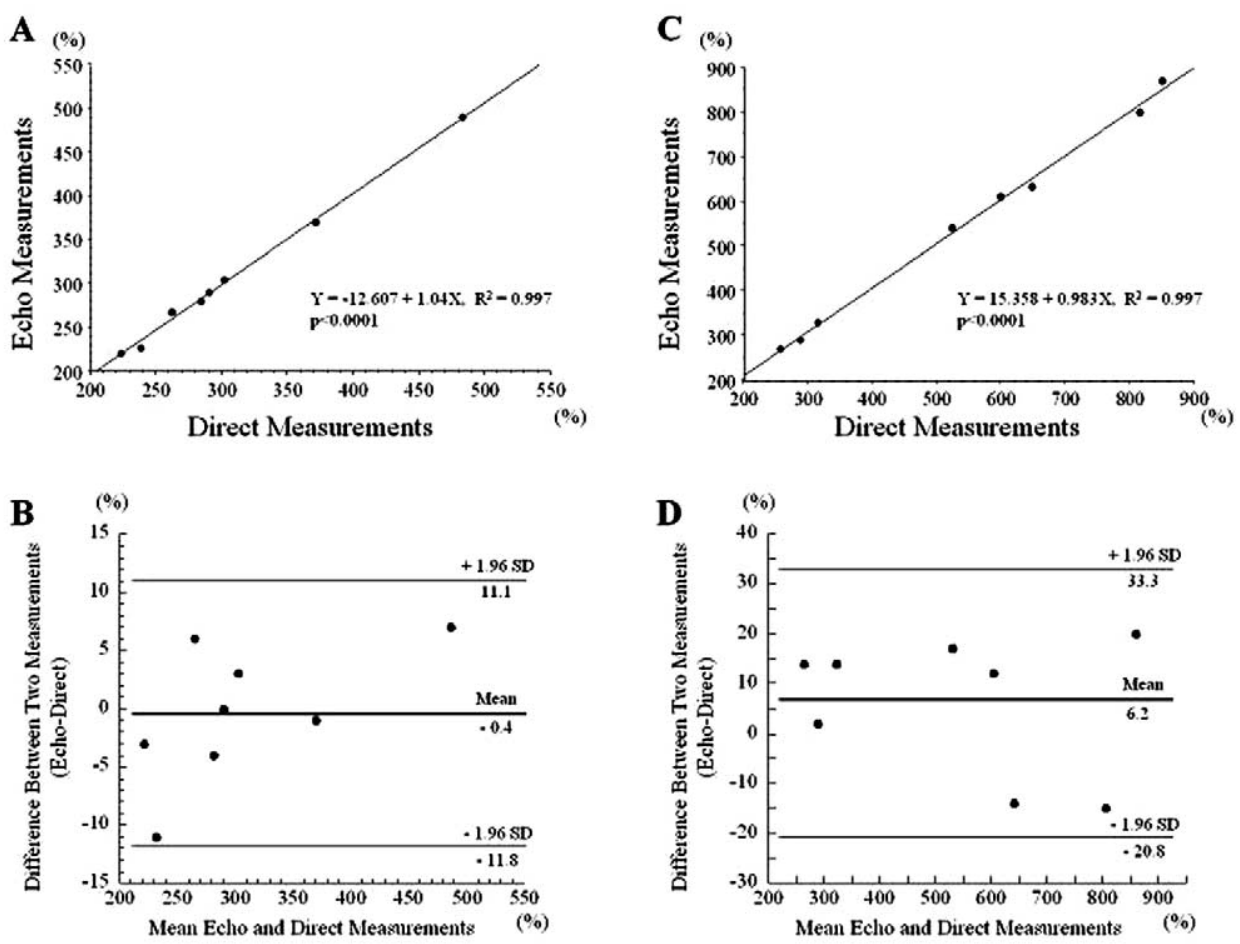

Figure 5. Linear regression (A) and corresponding Bland-Altman plots (B) comparing percentage enlargement of ASD from RT3DE images with that obtained by direct measurements conducted in tank study. Linear regression (C) and corresponding Bland-Altman plots (D) comparing percentage enlargement of ASD from RT3DE images with that obtained by direct measurements conducted in animal study.

\section{Statistical Analysis}

Data are expressed as mean $\pm \mathrm{SD}$. The differences in the size of the ASD and in the grade of the atrial shunt before and after atrial septectomy were analyzed by Student $t$ test and Mann-Whitney test. Simple regression analysis was performed to calculate the correlation between the \%ERT3DE and \%EDM in the in vitro and in vivo experiments. Bland-Altman analysis was performed to further determine the agreement of the results between the two measurement methods. ${ }^{6}$ Statistical analysis was performed with the StatView software package (SAS Institute Inc, Cary, NC) and MedCalc software (MedCalc Software, Mariakerke, Belgium).

\section{Results}

\section{Tank Study}

Representative echocardiographic images obtained during the atrial septectomy are shown in Figure 3. Echo shadows produced on the surface of the atrial septal tissue by the Kerrison bone punch were beneficial for estimating the distance from the target. ASDs were significantly enlarged by atrial septectomy, from $27.2 \pm 7.1 \mathrm{~mm}^{2}$ before atrial septectomy to $79.8 \pm 15.9 \mathrm{~mm}^{2}$ after atrial septectomy $(P$ $<.0001$ ), as shown in Figure 4, $A$. The mean \%ERT3DE and $\%$ EDM were $307.0 \% \pm 84.2 \%$ and $306.2 \% \pm 87.8 \%$, respectively. There was a significant correlation between $\%$ ERT3DE and \%EDM $(\%$ ERT3DE $=-12.607 \pm 1.04 \times$
\%EDM, $P<.0001, \mathrm{n}=8$; Figure 5, A). Bland-Altman analysis showed excellent agreement between \%ERT3DE and \%EDM (difference $-0.4 \mathrm{~mm}^{2}$, upper and lower limits of agreement 11.1 and $-11.8 \mathrm{~mm}^{2}$, respectively; Figure 5, $B)$.

\section{Animal Study}

Representative echocardiographic images obtained during atrial septectomy in the animal study are shown in Figure 6. As seen in Figure 6, A, the spatial orientation of the ASD, the atrial septal tissue, other structures such as the tricuspid valve and the coronary sinus, and the Kerrison bone punch were easily recognized by RT3DE. Small ASDs created with the balloon catheter were significantly enlarged by atrial septectomy, from $22.6 \pm 7.9 \mathrm{~mm}^{2}$ before atrial septectomy to $101.5 \pm 27.8 \mathrm{~mm}^{2}$ after atrial septectomy $(P=$ .0001 ), as shown in Figure 4, $B$. The atrial shunt, as graded by 2-dimensional epicardial echocardiography, was significantly increased from $2.4 \pm 0.5$ before atrial septectomy to $4.7 \pm 0.5$ after atrial septectomy $(P<.0001$; Figure $7, A$ and $B)$. The mean \%ERT3DE and \%EDM were $543.3 \% \pm$ $229.8 \%$ and $537.2 \% \pm 233.5 \%$, respectively. The $\%$ ERT3DE was correlated with the \%EDM (\%ERT3DE = $15.358 \pm 0.983 \times \% \mathrm{EDM}, P<.0001, \mathrm{n}=8$; Figure $5, C)$. 
Bland-Altman analysis showed excellent agreement between \%ERT3DE and \%EDM (difference $6.2 \mathrm{~mm}^{2}$, upper and lower limits of agreement 33.3 and $-20.8 \mathrm{~mm}^{2}$, respectively; Figure 5, $D$ ). During the procedure, 3-dimensional echocardiography revealed no evidence of introduction of air or particulate emboli, and no tricuspid valve injury or heart block was observed. In addition, no significant blood loss was observed.

\section{Discussion}

Creation of an ASD for mixing oxygenated with deoxygenated blood through the atrial septum is necessary in the treatment of some types of complex congenital cardiac anomalies ${ }^{1}$. Although balloon atrial septostomy or blade septostomy followed by dilatation has been the standard approach until now for these patients, ${ }^{3,7}$ these methods prove to be extremely difficult in some cases, especially in older infants. On the other hand, the Blalock-Hanlon operation can be performed to directly create an ASD, but performance of this procedure requires expert technical skill, ${ }^{4}$ and control of the ASD diameter and shunt size can be extremely difficult. Although surgical atrial septectomy with cardiopulmonary bypass may by far be the most effective method, cardiopulmonary bypass itself is a major intervention, especially in critically ill patients.

Kawahira and colleagues ${ }^{8}$ have previously reported successfully performing a surgical atrial septostomy with an atriotomy knife without cardiopulmonary bypass. Takahashi and associates ${ }^{9}$ also reported successful creation of an ASD in a patient with transposition of the great arteries with a device similar to the one used in this study. Both groups used conventional cross-sectional epicardiography or transesophageal echocardiography to obtain guidance during the procedures. However, these imaging methods do not permit full evaluation of the precise spatial relationship between the foramen ovale and other structures, resulting in the possibility of injury to cardiac tissue or creation of too large an ASD. In this study we were able to create an ASD of appropriate size in a step-by-step manner under RT3DE guidance in the animal experiment, and we believe that this imaging method may be more effective than the previously used methods.

A variety of 3-dimensional echocardiographic systems have been developed during the past decade to provide clinicians and surgeons with a new perspective for visualizing the heart. ${ }^{10,11}$ Nevertheless, the current off-line system of 3-dimensional reconstruction from multiple cross-sectional echocardiographic scans is time-consuming, and this process requires a substantial learning curve. ${ }^{12}$ RT3DE in particular serves as a noninvasive method for real-time visualization of the heart without electrocardiographic or respiratory gating, because all planes are imaged simultaneously. ${ }^{5}$ Accordingly, we used this new methodology,
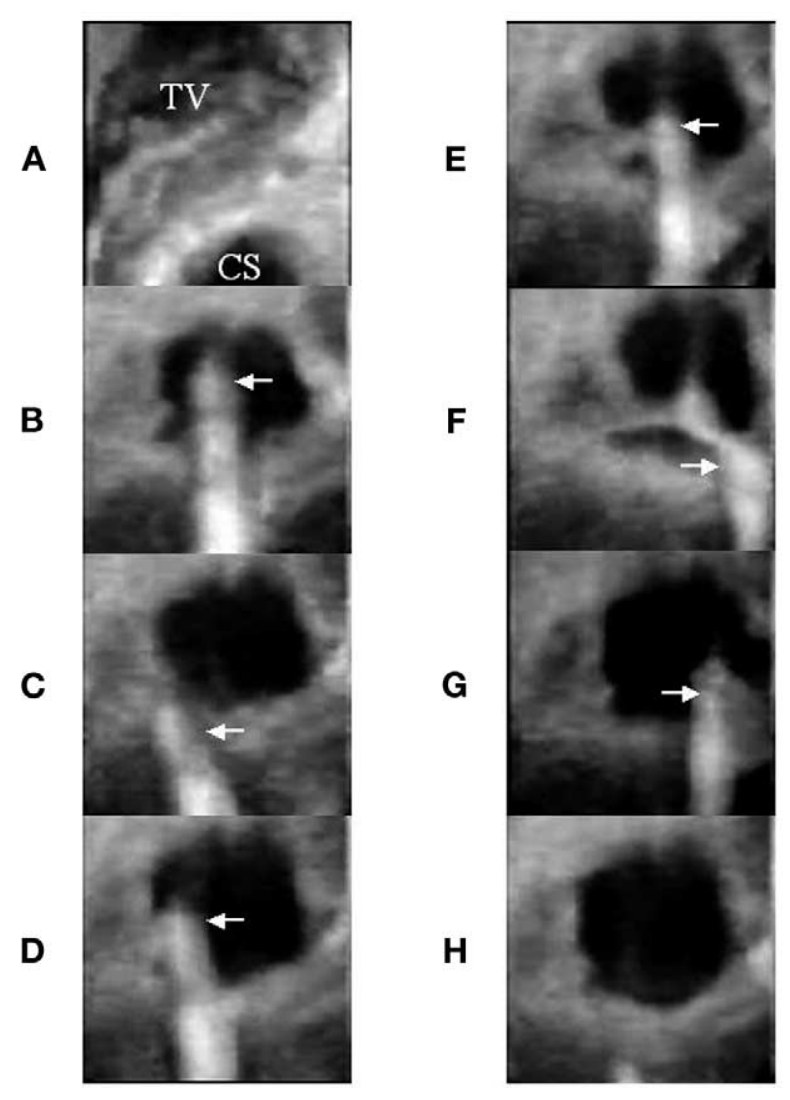

Figure 6. RT3DE images from animal study showing presurgical evaluation of structures inside heart (A) and atrial septectomy with Kerrison bone punch (B-H). White arrow indicates tip of Kerrison bone punch. $T V$, Tricuspid valve; $C S$, coronary sinus.

which can generate images with sufficient spatial and temporal resolution, as the sole guiding system for beating-heart surgery.

We used two different RT3DE systems in this study. One was a full volume-rendering system with a 2-dimensional matrix array transducer, and the other was a high-speed volume-rendering system with a mechanical 1-dimensional microconvex array transducer. Both the RT3DE systems, used in different experimental settings, yielded satisfactory images. Both have advantages and disadvantages with respect to the transducer size, frame rate, and frequency field of the transducer. For the animal experiment, we used the latter of the two aforementioned systems because of its small transducer size and high frequency field, despite the low frame rate. However, this system would be suboptimal for the visualization of rapidly moving structures, such as the heart valves, and the former of the two systems was therefore deemed to be more suitable. Further technologic advances of the RT3DE system, such as the development of a high-frequency 2-dimensional matrix minitransducer or transesophageal transducer, are desirable. 

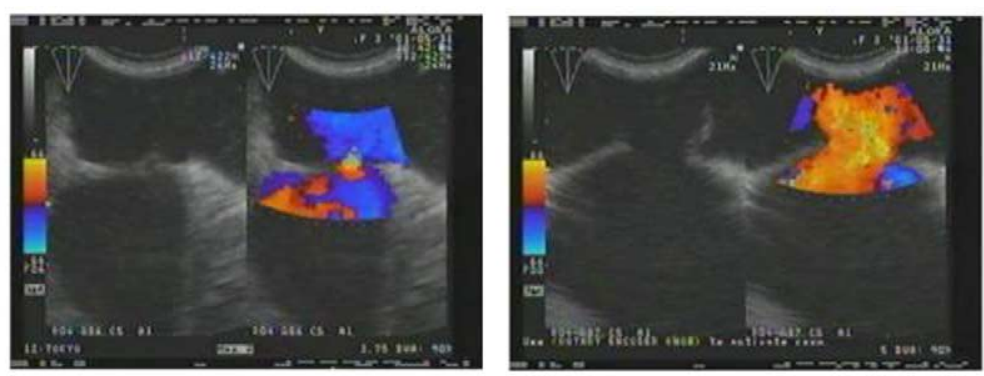

A

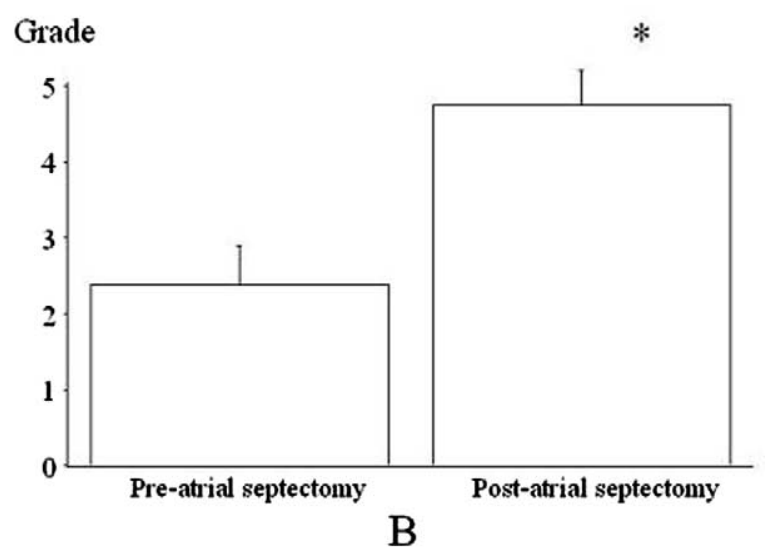

Figure 7. A, Color Doppler images obtained by 2-dimensional echocardiography before (left) and after (right) atrial septectomy. B, Atrial shunt score from 1 to 5 graded by 2-dimensional echocardiography. Significant difference at $P<.05$ versus size of ASD before atrial septectomy is designated by an asterisk.

Although 3-dimensional computed tomographic and magnetic resonance imaging methods may provide highresolution images of the heart, both involve prolonged imaging time, high equipment costs, and special facilities. The lack of portability of such systems also limits their clinical use in the operating room. Furthermore, the metallic contents of advanced medical devices that are occasionally used for operations preclude the use of these imaging systems, especially magnetic resonance imaging ${ }^{13}$. By contrast, these limitations can be overcome with the RT3DE systems used by us because of the lower cost, better portability, easier access, and absence of risk of radiation exposure.

\section{Limitations of the Study}

This study has several limitations. First, the surgical devices used did not have surface coating to minimize echo artifacts. Metallic devices sometimes produce severe artifacts and image distortion during echocardiographic imaging. Therefore future development of surface coatings for metallic devices or instrument designs compatible with echocardiographic imaging would be beneficial for 3-dimensional echocardiography-guided surgery. Second, we examined the percentage increase in the size of the ASDs for the validation study of the 3-dimensional images because the Sonos 7500 system is not equipped with a measurement system or software. In the future it will be necessary to develop measurement software for the RT3DE system and conduct a validation study of that system.

\section{Conclusion}

In summary, two different RT3DE systems were used in this study, and both were found to provide satisfactory images and sufficient anatomic detail to allow their use as the sole guidance system for surgical atrial septectomy. RT3DEguided atrial septectomy is expected to make the creation of an ASD safer and easier than with other surgical options. These results provide encouragement for the clinical application of RT3DE-guided atrial septectomy in selected patients, although we still need further studies to establish the safety of this procedure.

\section{References}

1. Sreeram N, Walsh K, Nobre A, Smith A, Peart I, Arnold R. Absent left-sided atrioventricular connexion, with right atrium connected to 
left ventricle: prospective diagnosis in infancy, and outcome. Int J Cardiol. 1992;34:7-19.

2. Rashkind WJ, Miller WW. Creation of an atrial septal defect without thoracotomy. A palliative approach to complete transposition of the great arteries. JAMA. 1966;196:991-2.

3. Webber SA, Culham JA, Sandor GG, Patterson MW. Balloon dilatation of restrictive interatrial communications in congenital heart disease. Br Heart J. 1991;65:346-8.

4. Blalock A, Hanlon CR. The surgical treatment of complete transposition of the aorta and pulmonary artery. Surg Gynecol Obstet. 1950; 90:1-15.

5. Sugeng L, Weinert L, Thiele K, Lang RM. Real-time three-dimensional echocardiography using a novel matrix array transducer. Echocardiography. 2003;20:623-35.

6. Bland JM, Altman DG. Statistical methods for assessing agreement between two methods of clinical measurement. Lancet. 1986;1:307-10.

7. Park SC, Neches WH, Zuberbuhler JR, Lenox CC, Mathews RA, Fricker FJ, et al. Clinical use of blade atrial septostomy. Circulation. 1978;58:600-6.
8. Kawahira Y, Kishimoto H, Kawata H, Ikawa S, Ueda H, Ueno T, et al. Surgical atrial septostomy without cardiopulmonary bypass. Ann Thorac Surg. 1996;61:1016-8.

9. Takahashi M, Kanazawa H, Yamazaki Y, Ueno M, Sakano T. A new, safe, and easy technique of atrial septal defect creation. Ann Thorac Surg. 2000;69:293-4.

10. Takuma S, Cardinale C, Homma S. Real-time three-dimensional stress echocardiography: a review of current applications. Echocardiography. 2000;17:791-4.

11. Lange A, Palka P, Burstow DJ, Godman MJ. Three-dimensional echocardiography: historical development and current applications. $J$ Am Soc Echocardiogr. 2001;14:403-12.

12. Vogel M, Ho SY, Lincoln C, Yacoub MH, Anderson RH. Threedimensional echocardiography can simulate intraoperative visualization of congenitally malformed hearts. Ann Thorac Surg. 1995;60: 1282-8.

13. Woodard EJ, Leon SP, Moriarty TM, Quinones A, Zamani AA, Jolesz FA. Initial experience with intraoperative magnetic resonance imaging in spine surgery. Spine. 2001;26:410-7. 